\title{
Intelligent Kite Aerial Platform for Site Photography
}

\author{
John C. Murray, Nark J. Neal, Frédéric Labrosse. \\ Department of Computer Science, \\ University of Wales, Aberystwyth, \\ Penglais Campus, \\ Aberystwyth, \\ Wales, SY23 3DB \\ \{jym,mjn,ffl\}@aber.ac.uk
}

\begin{abstract}
In this paper we present a semi-autonomous intelligent kite aerial photography platform (iKAPP). We detail the hardware and software aspects of the system and show how such a platform can be used to provide a portable, low cost, high quality solution to current aerial photography methods. In addition, we show how this system can be used to acquire high quality images which can be tiled together in order to create a high resolution detailed image of a large area. We use the system to acquire images of local sites of interest that have previously been photographed using other techniques and compare and present our results in this paper.
\end{abstract}

\section{BACKGROUND}

Kite aerial photography (KAP), a form of remote sensing, is not new in its application to site surveying. In fact, KAP is one of the oldest forms of remote sensing having been used for over 100 years. Using kites for the purpose of aerial photography has been about since the late 1800's and early 1900 's. One of the main uses of KAP in the early 1900's was its application to military reconnaissance. Not only were kites used to lift cameras for the use of taking photographs but larger 'Cody' kites famed for their lifting abilities (named after their creator Samuel Franklin Cody) were used to lift people into the air for surveillance purposes during the $1^{\text {st }}$ world war, allowing them to see over the horizon.

However, over the past several decades, with the increasing developments in technology and reducing costs of light aircraft and other technologies, KAP has become more of a tool for hobbyists and enthusiasts therefore taking a back seat for large scale aerial photography. Other aerial photography techniques are discussed in more detail in Section II.

In the past few years as camera technology is making way for lighter, smaller and higher quality cameras, thus kite aerial photography is becoming ever more popular amongst enthusiasts and, more recently, academics with many researchers taking an interest in KAP and its application to the surveying of sites of interest. In [1,3] J. S. Aber et al, show the application of KAP for monitoring the change in wetland vegetation. This kind of technology provides a very low cost solution to acquiring images to help with such research avoiding the need to use expensive as well as possibly polluting alternatives. KAP therefore provides a green solution to aerial photography [2].

Although the technology for cameras has improved dramatically in recent years with digital cameras being favored over the traditional film type, cameras currently used for KAP work tend to use wide angle lenses and therefore be used for taking large field of view images. In addition to this, the majority of current rigs tend to consist of statically positioned cameras with simple trigger mechanisms allowing the cameras to take an image at regular intervals. More advanced systems allow the user to control the position of the camera using servos and a radio control system. Although these systems provide more accurate control of the position of the camera they lack important feedback, thus preventing the operator from knowing exactly where the camera is pointing and thus preventing more accurate control over the system.

\section{Alternatives to KITE Aerial PHOTOGRAPHY}

There are several alternative methods to using kites for the acquisition aerial photographs, with each of these methods having their own advantages and disadvantages when compared to the KAP method. One alternative to KAP is the use of satellite technology for obtaining images. The main advantage to using satellite data for aerial image acquisition is that with internet tools such as Google earth and Google maps, much of the surface of the planet has been mapped to some level of detail.

At present satellite imaging can provide a resolution of $60 \mathrm{~cm}$ per pixel at a very high cost of $\$ 4000$ with an addition restriction being that some satellites have a delay of 20-30 days before they return back to the same point. As a requirement of the iKAPP platform is to be able to identify plants, this resolution is less than desirable.

Another, more preferred method of aerial photography is with the use of light aircraft. This generally involves fitting a camera to a small single manned aircraft or glider which then flies at a low altitude over the area of interest to acquire

Manuscript received May 18th, 2001. This work was sponsored by EPSRC under grant number EP/D058201/1. 
images. Using this method of aerial photography allows for large areas to be covered in a relatively short time period and in varying weather conditions. There are however limitations to using light aircraft. Firstly, there are restrictions on the minimum altitude of the aircraft with this being $300 \mathrm{~m}$ for built up areas and $150 \mathrm{~m}$ elsewhere. This restricts the detail and resolution in which wide angle lenses can capture. Also, there are many countries that have a lot of sites of archeological interest that do not give permission for light aircraft.

Unless photographing wide areas, this altitude is often less than ideal and presents a number of problems. For a start, atmospheric haze increases with altitude, thus affecting picture clarity. Also, if a smaller object such as a building or particular 'interesting' feature is being photographed, a telephoto lens must be used, requiring a higher shutter speed to prevent motion blur in the image, which in turn requires fast film that tends to be grainy and have poorer contrast.

There are also major costs issues associated with the use of light aircraft which include the cost of fuel, pilot and a plane hire. Finally, a more pressing problem is that if one wishes to acquire images of a restricted site, then prior permission may be required if there is a no-fly zone in place and of course this may be declined.

KAP overcomes these problems, as it provides a low cost solution to the acquisition of images due to the only energy requirements being battery power and wind. In addition, currently there are no restrictions on location in which one can fly a platform from a kite.

\section{THE IKAPP RIG}

Current KAP rigs are very limited in both their operational capabilities and in what they can achieve with the technology and cameras they utilize. The main drawback with current KAP rigs, as previously discussed, is that they provide little or no feedback to the operator as to the current view of the camera or the position of the servos/camera with relation to the horizon and the area being photographed. This therefore results in 'guess work' on the part of the user as to the image that will be taken by the rig. The downside of this is that the user will only know if the 'correct' image has been taken once the rig has been retrieved and the images analyzed.

\section{A. iKAPP Hardware}

The iKAPP rig presented in this paper overcomes the limitations, previously mentioned, of standard KAP rigs and also introduces many benefits and advantages to these conventional KAP platforms. The iKAPP platform is built around a fully functional computer system, therefore allowing some onboard processing.

The main hardware of the platform developed consists of a $633 \mathrm{MHz}$ complete mini-ITX mainboard, comprising a 40GB 2.5" hard disk drive with 512MB DDR Ram. This constitutes the main processing hardware for the platform, i.e. to control the peripherals, store images, run the control software and carry out any image and control processing required. This processing power gives the iKAPP rig the ability to perform many calculations and automate much of the image acquisition process.

The system provides full user feedback and operator control via a wireless connection from the platform to a ground station laptop. This allows for seamless connectivity between the platform and the operator so control can be left up to the platform or taken by the operator.

The iKAPP platform is equipped with two cameras as opposed to the traditional one used by other KAP systems. The first camera is a high resolution 3Mpix (2048x1536) Machine vision IEEE Firewire camera with a changeable CMount lens.

This camera is mounted on a servo controlled adjustable pan and tilt gimbal. The gimbal on the platform allows for the camera to move $110^{\circ}$ on the pan axis and $140^{\circ}$ on the tilt axis. Therefore, this enables the operator to position the camera to any desired angle within the range of the gimbal. The position of the main camera can either be controlled manually by using position sliders (shown in Fig. 1) or automatically by the platform using pre-programmed algorithms allowing for a scan type motion of the camera thus allowing for a large area of the site to be photographed. This gimbal movement provides the platform with greater flexibility over the area of ground of which images can be taken.

As this camera tends to be used to acquire very high detailed narrow images of the site it is sometimes difficult to know exactly what feature the camera is pointing at, therefore the iKAPP platform is equipped with a second low resolution static camera with a resolution of $640 \times 480$ pixels and a field-of-view (FOV) of $60^{\circ}$. Depending on the altitude of the platform this camera can provide a wide coverage of the surrounding area.

This camera also provides the operator with a large aerial view of the terrain directly beneath the iKAPP platform (see Fig. 1). This allows the operator to manually determine if there are particular features at the site which require further analysis or more detailed imaging. This is achieved positioning the main camera (using the sliders) towards the feature. Feedback is then provided by the system as can been seen in Fig. 1 with the use of a yellow bounding box showing the view of the main camera.

The platform measures $19 \mathrm{~cm} \times 31 \mathrm{~cm} \times 16 \mathrm{~cm}$ and weighs $1.8 \mathrm{Kg}$. The system is powered by an $11.1 \mathrm{~V} 3200 \mathrm{mAh} \mathrm{Li-}$ Poly battery, and provides a running time of 45 minutes. The main advantage to the iKAPP platform is its portability, with such a small footprint and light weight it can be easily packed into a backpack and taken where needed, easily deployed and then recovered, all for very low costs. Fig. 2 shows the iKAPP platform prototype with movable gimbal and high resolution camera. 


\section{B. Changeable Lenses}

The high resolution camera on the platform has changeable lenses, therefore allowing for variable field-ofview and zoom lenses to be attached depending on the particular application of the platform. The current lenses used by the platform we have developed include an $8 \mathrm{~mm}$, $25 \mathrm{~mm}$ and $50 \mathrm{~mm}$ lens. The uses of the various lenses are described in more detail in Section IV. Equation 1 gives the formula for determining the FOV of a particular lens.

$$
F O V=2 \times \tan ^{-1}(l /(2 \times f)),
$$

where $l$ is the length in $\mathrm{mm}$ of the side of the CCD that you wish to measure for FOV and $f$ is the focal length of the lens in $\mathrm{mm}$. The $50 \mathrm{~mm}$ lens used on the camera provides a $10^{\circ}$ FOV as shown by Equation 1 .

TABLE I

FIELD-OF-VIEW FOR DIFFERENT LENSES

\begin{tabular}{c|cc}
\hline \hline Focal Length(mm) & FOV H(deg) & FOV V(deg) \\
\hline 8 & 64.83 & 50.93 \\
25 & 22.97 & 17.33 \\
50 & 11.60 & 8.72 \\
\hline \hline
\end{tabular}

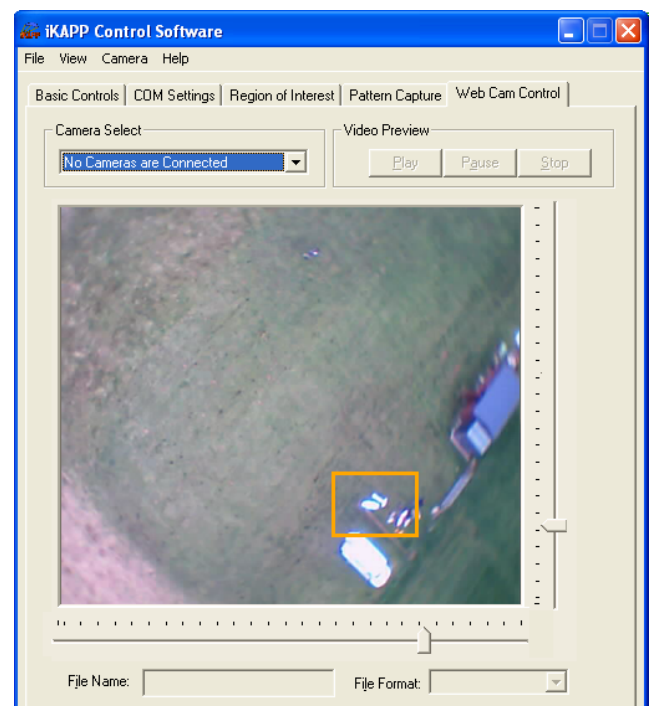

Fig. 1. WebCam GUI interface showing a wide FOV with box representing the current FOV of the HiRes camera.

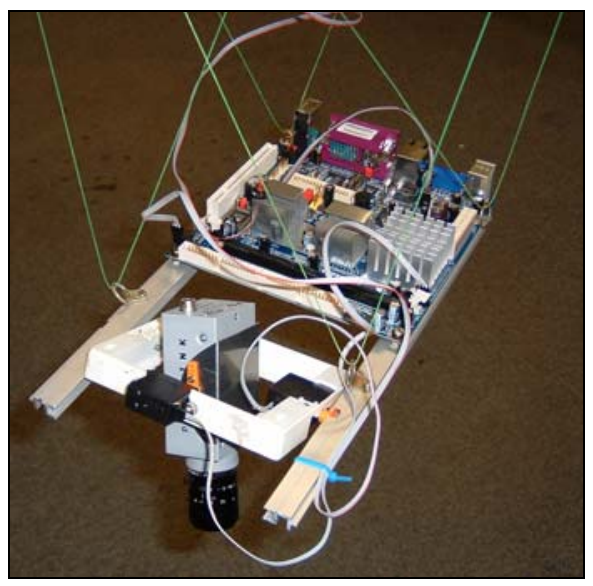

Fig. 3. The iKAPP 3 Platform prototype.

\section{C. iKAPP Control Software}

The system we present in this paper provides real-time feedback to the operator via the wireless connection and control software's interface. The software developed allows the operator to control all aspects of the iKAPP platform including the position of the pan and tilt gimbal, the rate of image capture in frames-per-second (FPS), brightness of the image filters, shutter speed of the Firewire camera, and the image capture type amongst other parameters.

Figure 3 shows the Pattern Capture panel of the control software. There are several control parameters that the operator needs to set; these include minimum and maximum pan and tilt values, pan and tilt movement increments and increment delay, i.e. how often to capture an image. These values are determined by the operator and are based on the current FOV of the lens being used on the camera (see Eq. 1) as it is important to allow the displacement value to move the gimbal just enough to create image overlap.

These values need to be considered carefully as the minimum and maximum pan and tilt values will determine the size of area of the site that is photographed whilst the pan and tilt increments will determine the offset of successive images as each of the images taken must include some degree of overlap to allow for a complete site map to be created (see Section V). The final parameter that needs to be set is 'capture delay' value, this is used to ensure that the movement of the camera gimbal has settled as much as possible before the image is taken, as this helps to reduce the possibility of motion blur within the image.

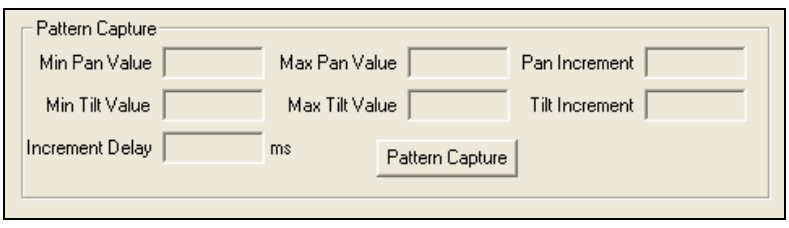

Fig. 3. Pattern capture GUI interface panel for iKAPP control software.

Figure 1 shows the webcam preview pane with an image of the terrain in the webcam view. The two sliders positioned along the two axis of the image are responsible for controlling the pan and tilt gimbal therefore allowing the main camera to point at a feature seen by the webcam. The current position of the main camera relative to the webcam is shown on the webcam image with a yellow box.

\section{ACQUiRING IMAGES}

In order to acquire the images of various sites, the iKAPP platform needs to be lifted above the site by a kite. Different kite designs have different attributes that make them suitable for lifting different weights in different wind conditions. For this project we have four different kite designs, each for use in varying wind conditions.

For the experimentation and results within this paper two of the four kites were used due to the varying wind conditions. These were the Dopero 100 and Sutton flow- 
form 30. Table II gives the dimensions and ideal wind conditions for each of the kites. For light winds of between $5 \mathrm{mph}-10 \mathrm{mph}$ we use the Dopero 100 . For stronger winds of up to $15+\mathrm{mph}$ the kite used is the Sutton flow-form 30 .

TABLE II

\begin{tabular}{|c|c|c|c|c|c|}
\hline \multirow[t]{2}{*}{ Kite } & \multicolumn{3}{|c|}{ Dimension } & \multirow{2}{*}{$\begin{array}{l}\text { Weight } \\
(\mathrm{Kg})\end{array}$} & \multirow{2}{*}{$\begin{array}{l}\text { Wind } \\
\text { Speed }\end{array}$} \\
\hline & $\mathrm{W}(\mathrm{m})$ & $\mathrm{H}(\mathrm{m})$ & Surface $(\mathrm{m} 2)$ & & \\
\hline $\begin{array}{c}\text { Dopero } \\
100\end{array}$ & 2.8 & 1.8 & 3.7 & 1 & Light \\
\hline $\begin{array}{c}\text { Flowform } \\
30\end{array}$ & 1.5 & 1.8 & 2.75 & 1 & $\begin{array}{l}\text { Light - } \\
\text { Medium }\end{array}$ \\
\hline $\begin{array}{c}\text { Cody } \\
\text { Boxkite }\end{array}$ & 2.43 & 1.42 & 2.2 & 1.3 & $\begin{array}{c}\text { Light - } \\
\text { Moderate }\end{array}$ \\
\hline
\end{tabular}

\section{A. Launching and Configuring the Platform}

When launching the kite it is necessary to release enough line to ensure that the kite gains the required altitude to ensure it finds a smooth air flow and thus a stable flight. The closer the kite to the ground the more unstable the air flow due to disturbances along the ground. Once the kite is stable, this tended to be at an altitude of $50 \mathrm{~m}$; the iKAPP platform is secured to the line and then lifted upwards by the kite.

The iKAPP platform is lifted to a height of approximately $40 \mathrm{~m}$. When stable the system is powered up and a remote desktop session is created and the operator can configure the platform. The initial configurations require setting the shutter speed of the camera. The brightness of the captured image depends on the lighting conditions and the shutter speed of the camera, as this is a machine vision camera the shutter speed refers to how long the pixels on the CCD remain active, the less light the longer they must remain active. Once this is configured the system is ready to begin acquiring images of the site.

\section{B. Choosing a Lens}

The application of the platform will decide on which particular lens is used for the task. In this paper, highly detailed images of the ground are captured and tiled to show how such images can be used to create larger maps of sites.

For the acquisition of images in this paper it was decided that the $25 \mathrm{~mm}$ lens be used, as this was best suited to foliage identification. This was due to the $8 \mathrm{~mm}$ lens providing a very wide angle of view (see Table I) and therefore not providing enough detail, and the $50 \mathrm{~mm}$ having too narrow a FOV covering just $5.3 \mathrm{~m}$ at a height of $30 \mathrm{~m}$ thus not creating enough image overlap. Figure 4 gives an example of the field of view of an image acquired by the $25 \mathrm{~mm}$ lens taken at a height of approximately $30 \mathrm{~m}$.

\section{REGISTERING THE IMAGES}

We have deployed the iKAPP platform at several sites around Aberystwyth, Wales, UK. In this paper we show the results of the image acquisition of one of the sites, namely that of Ystumtuen, Aberystwyth located at $52^{\circ} \mathrm{N}, 3^{\circ} \mathrm{W}$. Once the iKAPP platform was deployed at this site, the camera was configured and began capturing images of the site.

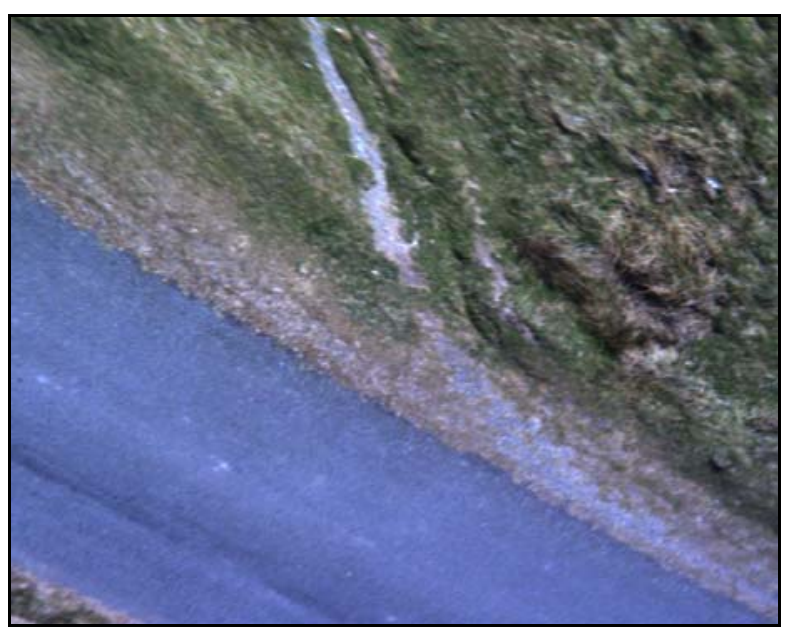

Fig. 4. An image showing a section of single lane road and grassland acquired with a $25 \mathrm{~mm}$ lens at a height of approximately $30 \mathrm{~m}$.

Over 150 high resolution images were captured of this site and later analyzed so as to tile them together to create a larger more complete image of the site. When creating a larger tiled image from the individually captured images, it is hoped that each of the images contains some overlap to another of the images. However, this is not entirely necessary in order to arrange the images into their correct positions as there are other ways to achieve this using the abilities of the platform.

This method involves using the static webcam. With each image capture made by the high resolution camera an image is also taken with the webcam. This therefore allows the high resolution, small FOV image to be compared against the low resolution, large FOV webcam image.

For purposes of demonstrating the platform in this paper, two sets of images in which overlap occurs are used to create two larger high resolution images. The first tile consists of eight individual images as is shown in Fig. 5 whilst the second tile also consists of eight images as shown in Fig. 6.

As Figures 5 and 6 show, the two sets of overlapped images form two large, high resolution, high detail regions of the site in question. The individual images used to create the tiles each have a resolution of $1280 \times 1024$. This resolution is smaller than is possible with the camera on the current iKAPP platform which is $2048 \times 1536$. However, it was decided to reduce the region-of-interest as to decrease the image capture time (see Section VI) therefore helping to reduce effects such as motion blur. The first tiled map combines eight images to create an image with a resolution of 6509x4199 at its widest points, Fig. 5.

Fig. 6 shows the combining of eight images to create a tiled map with a resolution of $2859 \times 2655$. As the images have been scaled down to display in this paper some quality and detail of the images has been lost. The original images can be seen on the project website:

http://www.ikapp.org/CASE07/images.php. 


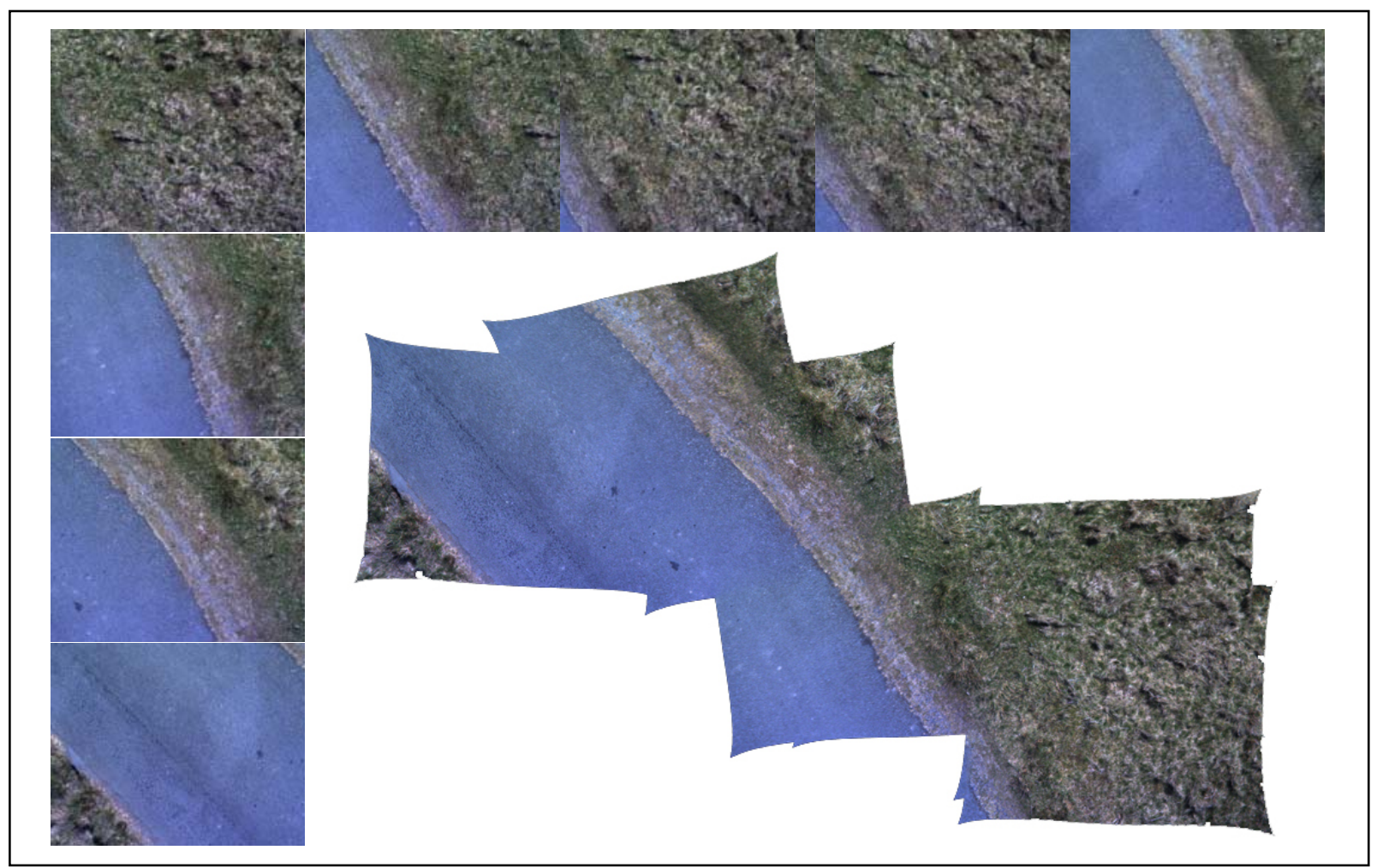

Fig. 5. Eight individual (overlapped) images tiled to create a higher resolution tiled image. Tiled image resolution: 6509x4199. Tiling of image performed by Software PTGui.

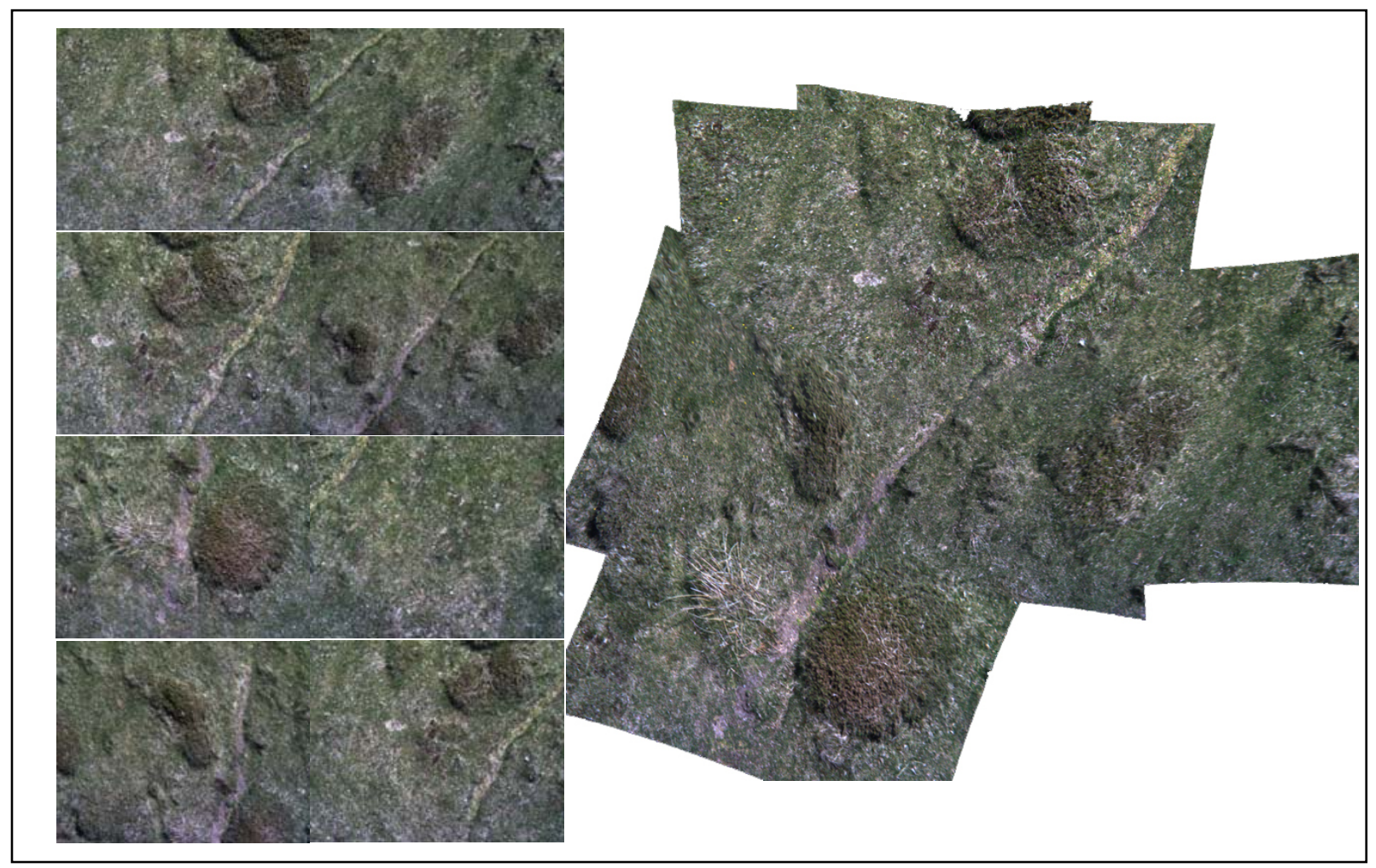

Fig. 6. Eight individual (overlapped) images tiled to create a higher resolution tiled image. Tiled image resolution: 2859x2655. Tiling of image performed by Software PTGui.

\section{DISCUSSION}

Looking closely at the images acquired of the features at the site, it can be seen that some of the images are not as clear and crisp as others. There are two main aspects of the system that cause the captured images to become blurred or out of focus. 
Acquisition of images for a particular site can take anywhere from $20-45$ minutes. Therefore, during this time due to the kites altitude and lifting capability being proportional to the current wind speed, the kite will vary in altitude. This change in altitude will affect the focus of the image. As the depth of field of a lens is set to a particular distance range, as the kite changes altitude the features will move in and out of this field of view boundary. Fig. 7 shows how within an image some of the features may be out of focus whilst some are in focus.

Another main cause of blurring in the images is well known in photography, that of motion blurring. The high resolution camera used on the iKAPP platform uses a rolling shutter (or push-broom) to sample the data from the CCD. Due to the platform being deployed in a very turbulent environment, this results in some of the captured images being subject to motion blurring. At the end of the exposure time the pixel values in the CCD are read out row-by-row. This begins as soon as the integration (exposure) time is complete. However, during the readout period, the pixels that have not yet been read are still sensitive and will continue to integrate and it can take up to $200 \mathrm{~ms}$ to read a full frame. Fig. 8 shows the effect of motion blurring in the images.

\section{FURTHER WORK}

There are several further advancements which are currently being applied to the iKAPP platform to improve both the abilities of the platform and the quality of the images acquired.

The task of registering the images is aided when the images overlap each other. As previously mentioned not all of the images acquired will contain this overlap, and so this is where the GPS data helps.

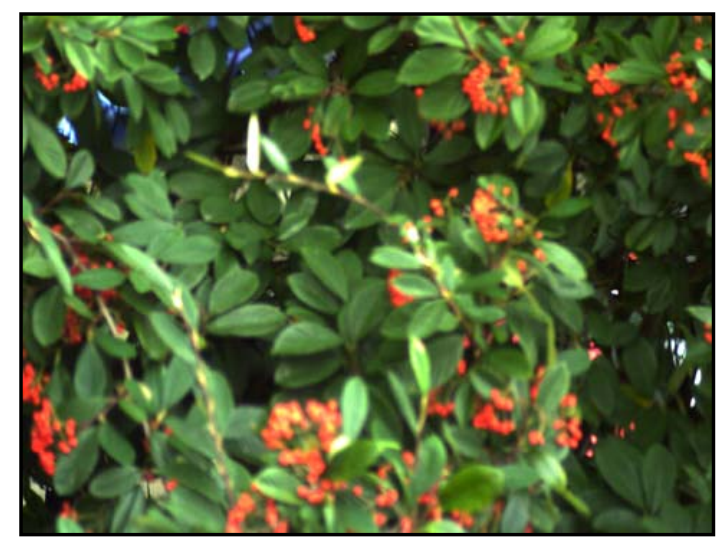

Fig. 7. Effects of depth of field causing focus blur.

However, the GPS data doesn't give us any orientation information about the platform and so it is difficult and sometimes impossible to know the orientation of the image in relation to other images. Therefore the platform is being equipped with a magnetometer to provide orientation information for the platform.

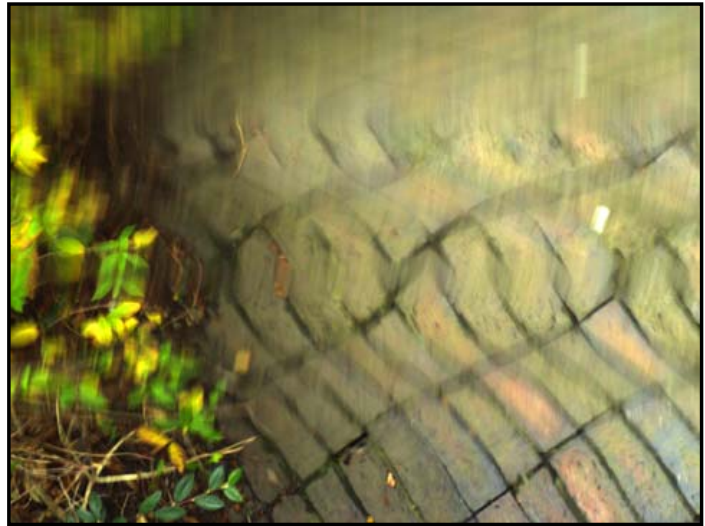

Fig. 8. Effects of motion blur on an image.

By using a kite for aerial photography and site surveillance, one is at the mercy of the direction of the wind to guide the kite. Multi-line kites (stunt kites) can be used to force a change in the position of the kite. However this is not a viable option for the system presented here as multiple lines have a tendency to become tangled and with a platform hanging down from the kite this introduces more problems.

As the kites used for KAP have a single line that branches off to multiple bridal lines close to the kite it may be possible to connect actuators to these lines that could be controlled by either a ground operator or the platform itself to control the direction of the kite.

To reduce motion blur in the images work is currently being carried out to introduce gyroscopic stabilization, provided by hardware gyros mounted on the platform and real-time software gyro stabilization.

\section{LAND, SEA AND AIR}

Combining the kite control with the GPS system it would be possible to plot the flight path that the aerial platform takes. In addition, each of the images captured can be tagged with their GPS locations.

However, within the department we have recently purchased two new pieces of equipment, namely a $3.65 \mathrm{~m}$ autonomous sailing boat and a large $1.3 \mathrm{~m} \times 2.3 \mathrm{~m}$ rover. As these weigh in excess of $100 \mathrm{Kg}$ each it would be possible to attach the kite to either the robot or the sailing boat and using wireless create an ad hoc network between the devices allowing fully autonomous land, sea and air surveillance.

\section{REFERENCES}

[1] J. S. Aber, S. W. Aber, F. Pavri, E. Volkova and R. L. Penner II, "Small-format aerial photography for assessing change in wetland vegetation, Cheyenne Bottoms, Kansas", in Transactions of the Kansas Academy of Science, vol. 109, issue 1-2, pp. 47-57, 2006.

[2] "Special Report - Green Technologies", in EPSRC Newsline, issue 37, pp. 2-3, 2006. Available at: http://www.epsrc.ac.uk/Publications /Newsline/Newsline37.htm

[3] J. S. Aber and D. Galazka, "Potential of kite aerial photography for Quaternary investigations in Poland", in Geological Quarterly, vol. 44,pp. 33-38, 2000. 